\title{
On the Structural Nature of Cooperation in Distributed Network Localization
}

\author{
Alireza Ghods, Stefano Severi and Giuseppe Abreu \\ Jacobs University Bremen \\ Campus Ring 1, 28759 Bremen \\ [a.ghods, s.severi,g.abreu] @jacobs-university.de
}

\author{
Samuel Van de Velde and Heidi Steendam \\ Ghent University \\ Sint-Pietersnieuwstr, 25, 9000, Ghent \\ slvdveldatelin.ugent.be
}

\begin{abstract}
We demonstrate analytically that the contribution of cooperation in improving the accuracy of distributed network localization has a fundamentally structural nature, rather then statistical as widely believed. To this end we first introduce a new approach to build Fisher Information Matrices (FIMs), in which the individual contribution of each cooperative pair of nodes is captured explicitly by a corresponding information vector. The approach offers new insight onto the structure of FIMs, enabling us to easily account for both anchor and node location uncertainties in assessing lower bounds on localization errors. Using this construction it is surprisingly found that in the presence of node location uncertainty and regardless of ranging error variances or network size, the Fisher information matrix (FIM) terms corresponding to the information added by nodeto-node cooperation nearly vanish. In other words, the analysis reveals that the key contribution of cooperation in network localization is not to add statistical node-to-node information (in the Fisher sense), but rather to provide a structure over which information is better exploited.
\end{abstract}

\section{INTRODUCTION}

It has long been of common knowledge that cooperation is advantageous as a means to improve the accuracy of network localization systems [1]-[4]. However, despite the abundance of empirical evidence on the latter, and a good amount of analytical work on the analysis of the fundamental errors achievable by localization systems [5]-[7], little is known about the actual nature of the gains reaped by cooperation in a distributed setting. Indeed, to the best of our knowledge no analytical results exists that are applicable to distributed localization systems.

What distinguishes the distributed case from others is the following. In a centralized system, all the information is gathered at a common point and processed jointly in order to derive estimates for the location of all nodes simultaneously. Consequently, the only errors the estimators are subject to are extrinsic, that is, the errors of the (typically geometric) input provided, such as distance and angle of arrival (AoA) estimates. In contrast, distributed systems must rely on the successive exchange both of geometric input and previous location estimates amongst nodes, such that the fundamental limits of the centralized and distributed are bound to differ.

In lack of a clear understanding of the nature of the cooperative advantage in the distributed setting, a heuristic notion that cooperation improves performance by increasing the amount of available information (in a Fisher sense) - which is indeed true in the centralized case, but as shall be here demonstrated not so in the distributed setting - is only natural to arise.
Indeed, resting partly on such intuitive notion, and in view of the challenge in exploiting less accurate information (e.g., originating from links in non line-of-sight (NLOS) conditions) in practical algorithms, various techniques have been proposed to censor or select input data based precisely and mostly on the comparison of their statistical characteristics [8]-[10].

In this article, we demonstrate that contrary to such common understanding, the contribution of cooperation in improving the accuracy of distributed network localization systems is fundamentally structural, rather then statistical.

\section{Fisher INFORMATION MATRICES AND INFORMATION VECTORS}

\section{A. Notation and Definitions}

Consider a network of $K$ devices, out of which $n_{\mathrm{T}}$ are "target" nodes (hereafter simply referred to as nodes) of unknown locations, and $n_{\mathrm{A}}$ are anchors, assumed to have known positions, possibly subject to errors.

Throughout the article we will use the subscript $n$ exclusively to denote nodes, and $k$ to denote either nodes or anchors. Following this convention, let $\boldsymbol{\theta}_{n}$ and $\boldsymbol{\theta}_{k}$ respectively denote $\eta$-dimensional row vectors with the coordinates of a node and a node/anchor, and consider the functional mapping

$$
g\left(\boldsymbol{\theta}_{n} \mid \boldsymbol{\theta}_{k}\right): \mathbb{R}^{\eta} \longrightarrow \mathbb{R}
$$

For any given pair $(n, k)$, the function $g\left(\boldsymbol{\theta}_{n} \mid \boldsymbol{\theta}_{k}\right)$ corresponds to a measure that captures the distinction (or dissimilarity) between the node $n$ and the node/anchor $k$. Consequently, we shall refer to $g(\cdot \mid \cdot)$ as the dissimilarity function.

In the context of network localization, the output of $g(\cdot \mid \cdot)$ may be either geometric - e.g., Euclidean distances [11] or angle information [12] [13] - or any other measurable physical parameter such as receive signal strength [14] [15], time of arrival [16] [17] [18], etc. Furthermore, each pair $(k, n)$ can in principle have multiple variation functions. For the sake of simplicity, however, we will assume hereafter (without a fundamental loss of generality) that a single dissimilarity is collected for each pair $(k, n)$.

A given dissimilarity function outputs the true value of the dissimilarity in question. Such dissimilarity is, however, measured subject to errors of statistics modelled by an associated probability density function (PDF), namely,

$$
p_{k n}\left(r_{k n} ; g\left(\boldsymbol{\theta}_{n} \mid \boldsymbol{\theta}_{k}\right)\right) \text {. }
$$


Notice that $g(\cdot \mid \cdot)$ defines the type of each input $r_{k n}$ used for localization purposes, while the densities $p_{k n}$ describe the variation of its observation at a specific pair, due to various random effects such as noise, mobility, etc. We shall therefore refer to $p_{k n}$ as the variation function.

\section{B. Information Vectors}

Define the vector $\boldsymbol{\theta} \triangleq\left[\boldsymbol{\theta}_{1}, \cdots, \boldsymbol{\theta}_{n_{\mathrm{T}}}\right]$ including the coordinate of all the targets in the network. Under the aforementioned assumptions, there are in total ${ }^{1} N \triangleq \frac{n_{\mathrm{T}}}{2}\left(2 K-n_{\mathrm{T}}-1\right)$ distinct and independent dissimilarities to be used in the localization problem, which are represented by the vector of variables $\mathbf{r} \triangleq\left[r_{21}, \cdots, r_{K 1}, r_{32}, \cdots, r_{3 K}, \cdots, r_{n_{\mathrm{T}} K}\right]$.

Let us assume that the vector $\boldsymbol{\theta}$ can be estimated by an unbiased estimator. Under the independence assumption, the associated Fisher information matrix (FIM) can be obtained from the following likelihood function

$$
\mathcal{L}(\mathbf{r} \mid \boldsymbol{\theta})=\prod_{n=1}^{n_{\mathrm{T}}} \prod_{k=n+1}^{K} p_{k n}\left(r_{k n} ; g\left(\boldsymbol{\theta}_{n} \mid \boldsymbol{\theta}_{k}\right)\right),
$$

yielding

$$
\mathbf{F}_{\boldsymbol{\theta}} \triangleq \mathbb{E}\left[\nabla_{\boldsymbol{\theta}} \ln (\mathcal{L}(\mathbf{r} \mid \boldsymbol{\theta})) \nabla_{\boldsymbol{\theta}}^{\mathrm{T}} \ln (\mathcal{L}(\mathbf{r} \mid \boldsymbol{\theta}))\right],
$$

where $\mathbb{E}[\cdot]$ denotes the expectation over the distribution of the input and $\nabla_{\boldsymbol{\theta}}$ is the gradient operator along the vector $\boldsymbol{\theta}$.

It is well-known [19] that the covariance matrix corresponding to the unbiased estimates $\hat{\boldsymbol{\theta}}$ is bounded by the Cramèr-Rao lower bound (CRLB)

$$
\boldsymbol{\Omega}_{\boldsymbol{\theta}} \triangleq \mathbb{E}\left[(\hat{\boldsymbol{\theta}}-\boldsymbol{\theta})(\hat{\boldsymbol{\theta}}-\boldsymbol{\theta})^{\mathrm{T}}\right] \succeq \mathbf{F}_{\boldsymbol{\theta}}^{-1}
$$

where the symbol $\succeq$ denotes positive-semidefinite inequality and ${ }^{-1}$ denotes inverse (or pseudo-inverse).

Let us now introduce the following result about $\mathbf{F}_{\boldsymbol{\theta}}$.

\section{Lemma 1 (Sum-product Formulation of FIM).}

Consider the unbiased estimation of $\boldsymbol{\theta}$ from the input vector $\mathbf{r}$ with associated dissimilarity functions $g\left(\boldsymbol{\theta}_{n} \mid \boldsymbol{\theta}_{k}\right)$ and variation functions $p_{k n}\left(r_{k n} ; g\left(\boldsymbol{\theta}_{n} \mid \boldsymbol{\theta}_{k}\right)\right)$, where $n=\left\{1, \cdots, n_{\mathrm{T}}\right\}$ and $k=\{1, \cdots, K\}$. Define the information vector related to each independent input $r_{k n}$ as

$$
\mathbf{v}_{k n} \triangleq \frac{\partial g\left(\boldsymbol{\theta}_{n} \mid \boldsymbol{\theta}_{k}\right)}{\partial \boldsymbol{\theta}} \sqrt{F_{k n}}
$$

where $F_{k n}$ is the Fisher information of each $r_{k n}$, given by

$$
F_{k n} \triangleq \mathbb{E}\left[\left(\frac{\partial \ln p_{k n}\left(r_{k n} ; g\left(\boldsymbol{\theta}_{n} \mid \boldsymbol{\theta}_{k}\right)\right)}{\partial g(\boldsymbol{\theta})}\right)^{2}\right] .
$$

Then $\mathbf{F}_{\boldsymbol{\theta}}$ can be conveniently obtained from the sum of information vector products

$$
\mathbf{F}=\sum_{n=1}^{n_{\mathrm{T}}} \sum_{k=n+1}^{K} \mathbf{v}_{k n} \mathbf{v}_{k n}^{T} .
$$

\footnotetext{
${ }^{1}$ Dissimilarities amongst anchors are considered to be constant and therefore are not included.
}

Proof: By definition - see equation (4) - we can rewrite

$$
\mathbf{F}_{\boldsymbol{\theta}}=\mathbb{E}\left[\sum_{k n} \sum_{i j} \mathbf{U}_{k n}\left(r_{k n} \mid \boldsymbol{\theta}\right) \mathbf{U}_{i j}^{\mathrm{T}}\left(r_{i j} \mid \boldsymbol{\theta}\right)\right],
$$

where $\mathbf{U}_{k n}\left(r_{k n} \mid \boldsymbol{\theta}\right)$ is known as the score function [20] of the random variables $r_{k n}$, defined by

$$
\mathbf{U}_{k n}\left(r_{k n} \mid \boldsymbol{\theta}\right) \triangleq \nabla_{\boldsymbol{\theta}} \ln \left(p_{k n}\left(r_{k n} ; g\left(\boldsymbol{\theta}_{n} \mid \boldsymbol{\theta}_{k}\right)\right)\right) .
$$

For an unbiased estimator, it is known [20] that the score functions have zero mean:

$$
\left.\mathbb{E}_{\tilde{d}_{k n}}\left[\mathbf{U}_{k n}\left(r_{k n} \mid \boldsymbol{\theta}\right)\right)\right]=0 .
$$

Using (11) into (9) and exploiting the independence between the input variables $r_{k n}$, it follows that

$$
\begin{aligned}
\mathbf{F}_{\boldsymbol{\theta}}= & \sum_{k n} \mathbb{E}\left[\mathbf{U}_{k n}\left(r_{k n} \mid \boldsymbol{\theta}\right) \mathbf{U}_{k n}^{\mathrm{T}}\left(r_{k n} \mid \boldsymbol{\theta}\right)\right] \\
& +\sum_{\substack{k n, i j \\
k n \neq i j}} \mathbb{E}\left[\mathbf{U}_{k n}\left(r_{k n} \mid \boldsymbol{\theta}\right)\right] \mathbb{E}_{\tilde{d}_{i j}}\left[\mathbf{U}_{i j}^{\mathrm{T}}\left(r_{i j} \mid \boldsymbol{\theta}\right)\right], \\
= & \sum_{k n} \mathbb{E}\left[\mathbf{U}_{k n}\left(r_{k n} \mid \boldsymbol{\theta}\right) \mathbf{U}_{k n}^{\mathrm{T}}\left(r_{k n} \mid \boldsymbol{\theta}\right)\right] .
\end{aligned}
$$

Using the chain rule we now rewrite the score function as

$$
\mathbf{U}_{k n}\left(r_{k n} \mid \boldsymbol{\theta}\right)=\frac{\partial \ln p_{k n}\left(r_{k n} ; g\left(\boldsymbol{\theta}_{n} \mid \boldsymbol{\theta}_{k}\right)\right)}{\partial g\left(\boldsymbol{\theta}_{n} \mid \boldsymbol{\theta}_{k}\right)} \frac{\partial g\left(\boldsymbol{\theta}_{n} \mid \boldsymbol{\theta}_{k}\right)}{\partial \boldsymbol{\theta}} \text {. }
$$

Substituting equation (6) and (13) into equation (12) yields

$$
\begin{aligned}
\mathbf{F}_{\boldsymbol{\theta}} & =\sum_{n k} \frac{\partial g(\boldsymbol{\theta})}{\partial \boldsymbol{\theta}} \mathbb{E}\left[\left(\frac{\partial \ln p_{k n}\left(r_{k n} ; g\left(\boldsymbol{\theta}_{n} \mid \boldsymbol{\theta}_{k}\right)\right)}{\partial g(\boldsymbol{\theta})}\right)^{2}\right] \frac{\partial g(\boldsymbol{\theta})^{\mathrm{T}}}{\partial \boldsymbol{\theta}} \\
& =\sum_{n=1}^{n_{\mathrm{T}}} \sum_{k=n+1}^{K} \mathbf{v}_{k n} \mathbf{v}_{k n}^{\mathrm{T}},
\end{aligned}
$$

which concludes the proof after defining the quantities in equations (6) and (7).

Notice that this formulation of the FIM is a contribution in its own right as it elucidates the effect of each independent input variable, in addition to being fully general on the input, in the sense that both $g\left(\boldsymbol{\theta}_{n} \mid \boldsymbol{\theta}_{k}\right)$ and $p_{k n}\left(r_{k n} ; g\left(\boldsymbol{\theta}_{n} \mid \boldsymbol{\theta}_{k}\right)\right)$ are arbitrary, except for independence. In the next section we shall however go a step further and show how the gain in insight provided by Lemma 1 can be exploited to achieve a better understanding of the fundamental nature of cooperation in the context of network localization.

\section{COOPERATION AND UNCERTAINTY IN DisTANCE-BASED NETWORK LOCALIZATION}

\section{A. Underlying Framework and Assumptions}

For the sake of illustration let us focus our analysis of cooperation in network localization on the particular - and predominant [11] [21] - case of distance-based approaches. In this case the dissimilarity function becomes

$$
g\left(\boldsymbol{\theta}_{n} \mid \boldsymbol{\theta}_{k}\right)=\left\|\boldsymbol{\theta}_{k}-\boldsymbol{\theta}_{n}\right\|=d_{k n} .
$$


Furthermore, in the interest of obtaining expressions that capture not only the variations incurred when measuring the distances $d_{k n}$, but also possible uncertainties on the location of anchors, we define the augmented (full network) coordinate vector

$$
\boldsymbol{\Theta} \triangleq\left[\boldsymbol{\theta}_{1}, \ldots, \boldsymbol{\theta}_{n_{\mathrm{T}}}, \boldsymbol{\theta}_{n_{\mathrm{T}+1}}, \ldots, \boldsymbol{\theta}_{K}\right]^{\mathrm{T}}
$$

such that the corresponding information vectors are given by

$$
\begin{aligned}
& \mathbf{v}_{k n}= \frac{\partial\left\|\boldsymbol{\theta}_{k}-\boldsymbol{\theta}_{n}\right\|}{\partial \boldsymbol{\Theta}} \sqrt{F_{k n}}= \\
&\left\{\begin{array}{lr}
{\left[\mathbf{0}_{1 \times \eta \cdot(n-1)}, \mathbf{u}_{k n}^{\mathrm{T}}, \mathbf{0}_{1 \times \eta \cdot(k-n-1)},-\mathbf{u}_{k n}^{\mathrm{T}}, \mathbf{0}_{1 \times \eta \cdot(K-k)}\right]^{\mathrm{T}},} \\
{\left[\mathbf{0}_{1 \times \eta \cdot K}\right]^{\mathrm{T}},} & \text { for } n \leq n_{\mathrm{T}},
\end{array}\right.
\end{aligned}
$$

where

$$
\mathbf{u}_{k n} \triangleq \frac{\partial\left\|\boldsymbol{\theta}_{k}-\boldsymbol{\theta}_{n}\right\|}{\partial \boldsymbol{\theta}_{n}} \sqrt{F_{k n}} .
$$

Equation (17) reveals the inherently sparse nature of the information vectors, which in light of Lemma 1 suggests that the corresponding FIM has a very rich structure. In order to evaluate such information vectors and FIM, however, specific underlying conditions must be specified.

For the sake of convenience, let us therefore limit ourselves (without fundamental loss of generality) to a two-dimensional scenario $(\eta=2)$, such that $\boldsymbol{\theta}_{n} \triangleq\left[x_{n}, y_{n}\right]$ and $\boldsymbol{\theta}_{k} \triangleq\left[x_{k}, y_{k}\right]$, which yields

$$
\mathbf{u}_{k n}=\frac{1}{d_{k n}}\left[x_{k}-x_{n}, y_{k}-y_{n}\right]^{\mathrm{T}} \sqrt{F_{k n}} .
$$

Furthermore, let us adopt the ranging model proposed in [18], which captures the fact that the accuracy of distance estimates obtained over a wireless channel depends, beyond a certain distance $d_{0}$, on the nominal distance themselves due to the unequal power with which signals are received. In this case, ranging errors can be modelled as normally-distributed variates with mean $d_{k n}$ and variance

$$
\sigma_{k n}^{2}=\left(\frac{d_{k n}}{d_{0}}\right)^{\alpha} \cdot \sigma_{0}^{2}
$$

where $\sigma_{0}^{2}$ is the variance at $d_{0}$ and with path-loss factor $\alpha \geq 0$.

In light of equation (15), the Fisher information associated with such a model can be shown to be given by [18]

$$
F_{k n}=\frac{1}{\sigma_{k n}^{2}}\left(1+\frac{\alpha^{2} \sigma_{0}^{2}}{2 d_{0}^{\alpha}} d_{k n}^{\alpha-2}\right)
$$

Using the models described above, and by force of Lemma 1 , the FIM of the entire network without a priory knowledge on anchor location uncertainty can be constructed as

$$
\mathbf{F}_{\Theta}=\sum_{n=1}^{K-1} \sum_{k=n+1}^{K} \mathbf{v}_{k n} \mathbf{v}_{k n}^{\mathrm{T}}
$$

\section{B. Anchor Location Uncertainties}

Let us assume that anchor location errors are independent and relatively small compared to deployment dimensions and ranging errors. In this case, anchor location uncertainty can be described by the block-diagonal covariance matrix

$$
\boldsymbol{\Sigma}=\operatorname{diag}\left(\boldsymbol{\Sigma}_{n_{\mathrm{T}}+1}, \cdots, \boldsymbol{\Sigma}_{K}\right),
$$

where each $\boldsymbol{\Sigma}_{k}, n_{\mathrm{T}+1} \leq k \leq K$ is an $\eta$-by- $\eta$ location error covariance matrix corresponding to a distinct anchor.

Under this assumption, the FIM associated with the estimate of $\Theta$ in presence of such anchor location uncertainty can be approximated - using the Bayesian rule - by

$$
\mathbf{F}_{\Theta}^{*} \approx \mathbf{F}_{\Theta}+\mathbf{F}_{\Sigma}
$$

where

$$
\mathbf{F}_{\boldsymbol{\Sigma}}=\left[\begin{array}{cc}
\mathbf{0}_{\eta n_{\mathrm{T}} \times \eta n_{\mathrm{T}}} & \mathbf{0}_{\eta n_{\mathrm{T}} \times \eta K} \\
\mathbf{0}_{K \eta \times \eta n_{\mathrm{T}}} & \boldsymbol{\Sigma}^{-1}
\end{array}\right] .
$$

Exploiting the structure of the information vectors described in equation (17), and using Lemma 1 as per equation (22), it can be shown that

$$
\mathbf{F}_{\boldsymbol{\Theta}}=\underbrace{\left[\begin{array}{cc}
\mathbf{A}_{\mathbf{1}} & -\mathbf{B}^{\mathrm{T}} \\
-\mathbf{B} & \mathbf{C}
\end{array}\right]}_{\text {node to anchor }}+\underbrace{\left[\begin{array}{cc}
\mathbf{A}_{\mathbf{2}} & \mathbf{0} \\
\mathbf{0} & \mathbf{0}
\end{array}\right]}_{\text {node to node }},
$$

with

$$
\begin{gathered}
\mathbf{A}_{\mathbf{1}} \triangleq \operatorname{diag}\left(\sum_{k=n_{\mathrm{T}+1}}^{K} \mathbf{u}_{k 1} \mathbf{u}_{k 1}^{\mathrm{T}}, \cdots, \sum_{k=n_{\mathrm{T}+1}}^{K} \mathbf{u}_{k n_{\mathrm{T}}} \mathbf{u}_{k n_{\mathrm{T}}}^{\mathrm{T}}\right), \\
\mathbf{C} \triangleq \operatorname{diag}\left(\sum_{n=1}^{n_{\mathrm{T}}} \mathbf{u}_{n_{\mathrm{T}+1} n} \mathbf{u}_{n_{\mathrm{T}+1}^{\mathrm{T}} n}^{\mathrm{T}}, \cdots, \sum_{n=1}^{n_{\mathrm{T}}} \mathbf{u}_{K n} \mathbf{u}_{K n}^{\mathrm{T}}\right), \\
\mathbf{B}^{\mathrm{T}} \triangleq\left[\begin{array}{cccc}
\mathbf{u}_{n_{\mathrm{T}+1} 1} \mathbf{u}_{n_{\mathrm{T}+1} 1}^{\mathrm{T}} & \mathbf{u}_{n_{\mathrm{T}+2} 1} \mathbf{u}_{n_{\mathrm{T}+2} 1}^{\mathrm{T}} & \ldots & \mathbf{u}_{K 1} \mathbf{u}_{K 1}^{\mathrm{T}} \\
\vdots & \vdots & \ddots & \vdots \\
\mathbf{u}_{n_{\mathrm{T}+1} n_{\mathrm{T}}} \mathbf{u}_{n_{\mathrm{T}+1} n_{\mathrm{T}}}^{\mathrm{T}} & \mathbf{u}_{n_{\mathrm{T}+2} n_{\mathrm{T}}} \mathbf{u}_{n_{\mathrm{T}+2} n_{\mathrm{T}}}^{\mathrm{T}} & \ldots & \mathbf{u}_{K n_{\mathrm{T}}} \mathbf{u}_{K n_{\mathrm{T}}}^{\mathrm{T}}
\end{array}\right],
\end{gathered}
$$

and

$$
\mathbf{A}_{\mathbf{2}} \triangleq\left[\begin{array}{cccc}
\sum_{k>1}^{n_{\mathrm{T}}} \mathbf{u}_{k 1} \mathbf{u}_{k 1}^{\mathrm{T}} & -\mathbf{u}_{21} \mathbf{u}_{21}^{\mathrm{T}} & \cdots & -\mathbf{u}_{n_{\mathrm{T}} 1} \mathbf{u}_{n_{\mathrm{T}} 1}^{\mathrm{T}} \\
-\mathbf{u}_{21} \mathbf{u}_{21}^{\mathrm{T}} & \mathbf{u}_{21} \mathbf{u}_{21}^{\mathrm{T}}+\sum_{k>2}^{n_{\mathrm{T}}} \mathbf{u}_{k 1} \mathbf{u}_{k 1}^{\mathrm{T}} & \ldots & -\mathbf{u}_{n_{\mathrm{T}} 2} \mathbf{u}_{n_{\mathrm{T}} 2}^{\mathrm{T}} \\
\vdots & \vdots & \ddots & \vdots \\
-\mathbf{u}_{n_{\mathrm{T}} 1} \mathbf{u}_{n_{\mathrm{T}} 1}^{\mathrm{T}} & -\mathbf{u}_{n_{\mathrm{T}} 2} \mathbf{u}_{n_{\mathrm{T}} 2}^{\mathrm{T}} & \cdots & \sum_{k=1}^{n_{\mathrm{T}}-1} \mathbf{u}_{n_{\mathrm{T}} k} \mathbf{u}_{n_{\mathrm{T}} k}^{\mathrm{T}}
\end{array}\right],
$$

where it is seen that no indices above $n_{\mathrm{T}}$ can be found in $\mathbf{A}_{\mathbf{2}}$.

Out of the above matrix, one is however typically interested in the errors on the estimates of node location ${ }^{2}$, i.e., $\boldsymbol{\theta}$. The FIM associated with $\boldsymbol{\theta}$ but accounting for anchor location uncertainty can be obtained from $\mathbf{F}_{\Theta}^{*}$ by taking its $\eta n_{\mathrm{T}} \times \eta n_{\mathrm{T}}$ Schur complement, namely

$$
\mathbf{F}_{\boldsymbol{\theta}}^{*}=\left(\mathbf{A}_{1}+\mathbf{A}_{2}\right)-\mathbf{B}^{\mathrm{T}}\left(\boldsymbol{\Sigma}^{-1}+\mathbf{C}\right)^{-1} \mathbf{B} .
$$

\footnotetext{
${ }^{2}$ This is because although anchors may be subject to location uncertainty, their positions are fixed and not repeatedly estimated as is the case of other nodes.
} 
Besides the FIM itself, the inverse of $\mathbf{F}_{\boldsymbol{\theta}}^{*}$ is of interest, as per equation (5). That can certainly be obtained numerically from equation (31), but in order to retain analytical insight let us consider the following pair of equalities

$$
\operatorname{tr}\left(\mathbf{F}_{\boldsymbol{\theta}}^{*}\right)=\sum_{i=1}^{\eta n_{\mathrm{T}}} \lambda_{i} \triangleq T \quad \text { and } \quad \operatorname{tr}\left(\mathbf{F}_{\boldsymbol{\theta}}^{*-1}\right)=\sum_{i=1}^{\eta n_{\mathrm{T}}} \frac{1}{\lambda_{i}},
$$

from which one can obtain

$$
\begin{gathered}
\frac{1}{T} \sum_{i=1}^{\eta n_{\mathrm{T}}} \lambda_{i}=1 \rightarrow \sum_{i=1}^{\eta n_{\mathrm{T}}} \frac{\lambda_{i}}{T}=1 \rightarrow \sum_{i=1}^{\eta n_{\mathrm{T}}} \lambda_{i}{ }^{\prime}=1, \\
\sum_{i=1}^{\eta n_{\mathrm{T}}} \frac{1}{\lambda_{i}{ }^{\prime}} \geq\left(\eta n_{\mathrm{T}}\right)^{2} \rightarrow \sum_{i=1}^{\eta n_{\mathrm{T}}} \frac{1}{\lambda_{i}} \geq \frac{\left(\eta n_{\mathrm{T}}\right)^{2}}{T},
\end{gathered}
$$

where we have used the geometric-arithmetic mean inequality.

Using the relations in (32) and (34) we finally obtain

$$
\frac{\operatorname{tr}\left(\mathbf{F}_{\boldsymbol{\theta}}^{*-1}\right)}{\eta n_{\mathrm{T}}} \geq \frac{\eta n_{\mathrm{T}}}{\operatorname{tr}\left(\mathbf{F}_{\boldsymbol{\theta}}^{*}\right)}
$$

which holds as an equality if and only if $\lambda_{i}=\lambda_{j}$ for $\forall i, j$.

From inequality (35) one concludes that in networks where all localization errors are similar - which tends to be the case in cooperative systems - the average CRLB can be well approximated by $\eta n_{\mathrm{T}} / \operatorname{tr}\left(\mathbf{F}_{\boldsymbol{\theta}}^{*}\right)$, such that the trace of the FIM itself is of great relevance.

Thanks to the structure revealed in equations (27) through (31), we obtain

$$
\begin{aligned}
\operatorname{tr}\left(\mathbf{F}_{\boldsymbol{\theta}}^{*}\right)= & \underbrace{\sum_{n=1}^{n_{\mathrm{T}}} \sum_{k=n_{\mathrm{T}+1}}^{K} \mathbf{u}_{k n} \mathbf{u}_{k n}^{\mathrm{T}}}_{\text {node to anchor }}+\underbrace{2 \sum_{n=1}^{n_{\mathrm{T}}-1} \sum_{k=n+1}^{n_{\mathrm{T}}} \mathbf{u}_{k n} \mathbf{u}_{k n}^{\mathrm{T}}}_{\text {node to node }} \\
& -\underbrace{\sum_{n=1}^{n_{\mathrm{T}}} \sum_{k=n_{\mathrm{T}+1}}^{K} \mathbf{u}_{k n} \mathbf{u}_{k n}^{\mathrm{T}}\left(\sum_{k}^{-1}+\sum_{i=1}^{n_{\mathrm{T}}} \mathbf{u}_{k n} \mathbf{u}_{k n}^{\mathrm{T}}\right)^{-1} \mathbf{u}_{k n} \mathbf{u}_{k n}^{\mathrm{T}}}_{\text {anchor uncertainty }},
\end{aligned}
$$

where we highlight the contributions of different structural components of the network.

Let us now turn our attention to the effect of the uncertainty of node locations themselves onto the cooperation that takes place in network localization.

\section{Node Location Uncertainty}

Notice that the FIM derived in equation (31) makes use only of the error distributions of measured distances, apart from the covariances of anchor location estimates. This implies that the corresponding CRLB applies only to centralized distance-based algorithms, in which location estimates of all nodes is obtained simultaneously, without relying on any prior information on the nodes' positions.

In distributed cooperative algorithms, on the other hand, nodes repeatedly exchange both ranging information and their own location estimates [1]-[3]. This indicates that the ultimate performance of such schemes must also dependent on the uncertainty on the exchanged node location estimates, which in turn are lower bounded by the CRLB and so on.

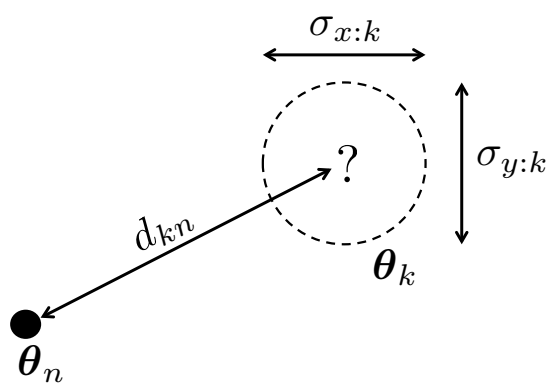

Fig. 1. Illustration of ranging process in the presence of node location uncertainty.

In other words, the fundamental performance limit of cooperative network localization systems can only be truly captured by the iterated posterior CRLB [22]. The problem with such an approach, however, is that only numerical results can be expressed, such that direct insight on the nature of the cooperation is hard to to be obtained.

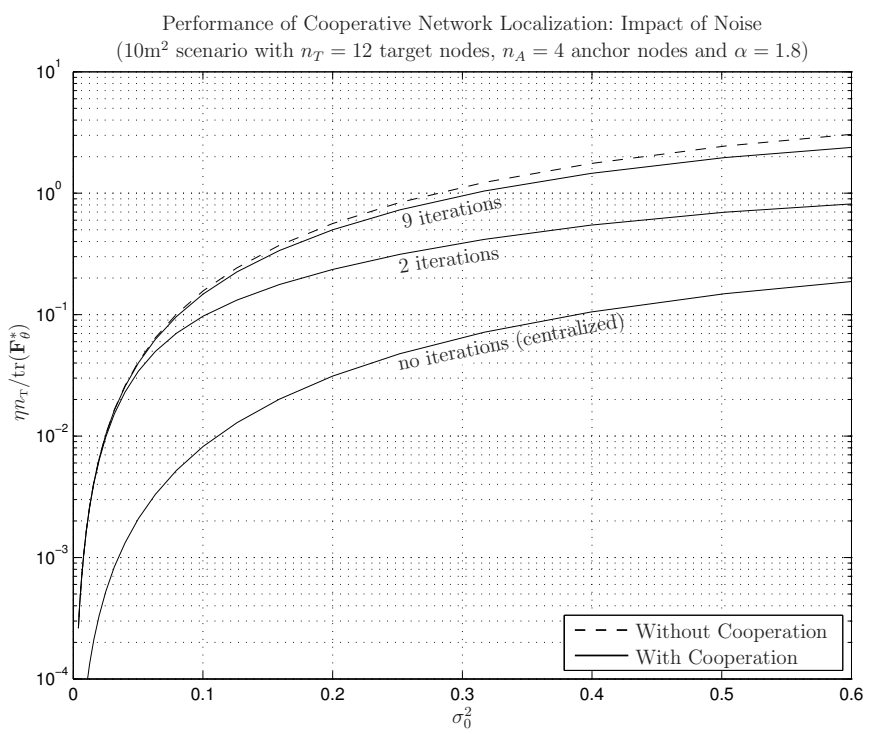

Fig. 2. Impact of node location uncertainty on the CRLB of cooperative network localization, as a function of reference ranging estimation errors.

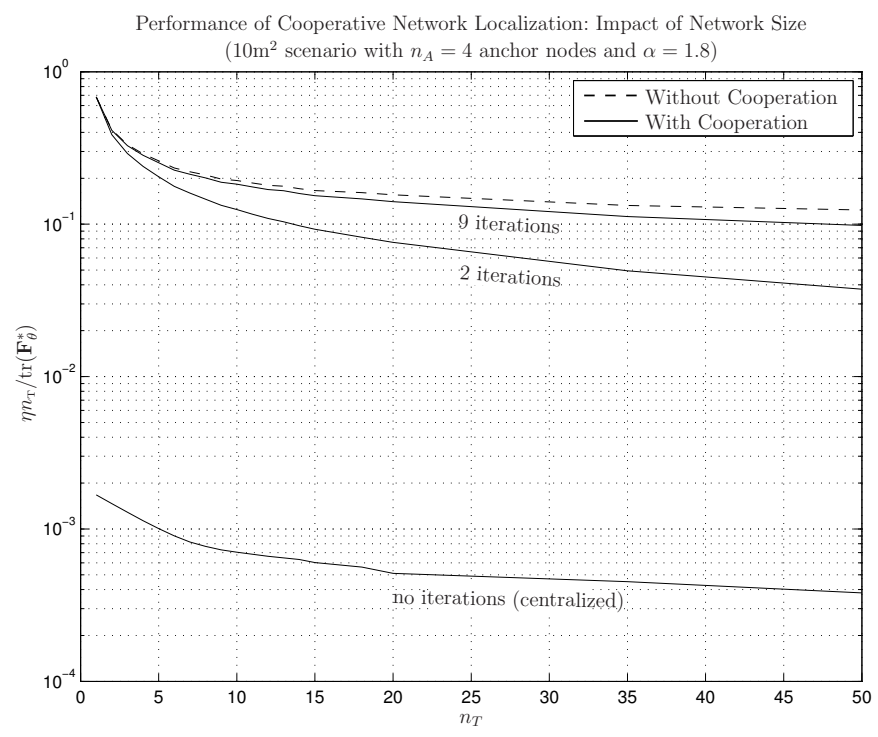

Fig. 3. Impact of node location uncertainty on the CRLB of cooperative network localization, as a function of the network size. 
In order to circumvent this problem, let us assume that the node location uncertainty is relatively small compared to the ranging errors. Let also $\sigma_{x: k}^{2}$ and $\sigma_{y: k}^{2}$ be the variances of the location estimate errors of the node at $\boldsymbol{\theta}_{k}$, along the $x$ and $y$ dimensions, respectively, such that the combined error variance along an unknown direction can be approximated by the quantity $\sigma_{d: k}^{2}=\frac{1}{2}\left(\sigma_{x: k}^{2}+\sigma_{y: k}^{2}\right)$, as illustrated in Figure 1. Between a pair of nodes $n$ and $k$, with $k \leq n_{\mathrm{T}}$, one can then consider that this additional uncertainty is transferred to the estimates of $d_{k n}$. Proceeding in this manner, the trace of $\mathbf{F}_{\theta}^{*}$ described in equation (36) and consequently the right hand side of inequality (35) can be evaluated iteratively. The results of such iteration are shown in Figures 2 and 3.

From these plots it can be appreciated that after only a few iterations, and regardless of either the reference ranging error variance $\sigma_{0}^{2}$ or the number of cooperating nodes $n_{\mathrm{T}}$, the lower bound on the CRLB gradually grows and converges approximately to the dashed curves shown in Figures 2 and 3 , which are obtaining by evaluating the right hand side of inequality (35) using a minorized version of $\operatorname{tr}\left(\mathbf{F}_{\boldsymbol{\theta}}^{*}\right)$, namely

$$
\begin{aligned}
\operatorname{tr}\left(\mathbf{F}_{\boldsymbol{\theta}}^{*}\right)= & \underbrace{\sum_{n=1}^{n_{\mathrm{T}}} \sum_{k=n_{\mathrm{T}+1}}^{K} \mathbf{u}_{k n} \mathbf{u}_{k n}^{\mathrm{T}}}_{\text {node to anchor }} \\
& -\underbrace{\sum_{n=1}^{n_{\mathrm{T}}} \sum_{k=n_{\mathrm{T}+1}}^{K} \mathbf{u}_{k n} \mathbf{u}_{k n}^{\mathrm{T}}\left(\Sigma_{k}^{-1}+\sum_{i=1}^{n_{\mathrm{T}}} \mathbf{u}_{k n} \mathbf{u}_{k n}^{\mathrm{T}}\right)^{-1} \mathbf{u}_{k n} \mathbf{u}_{k n}^{\mathrm{T}}}_{\text {anchor uncertainty }},
\end{aligned}
$$

which differs from equation (36) only in that the term related the cooperation amongst nodes has been removed.

In other words, the analysis indicates that the informational component of the cooperation has fundamentally no impact onto the achievable performance of cooperative network localization systems. The advantage of cooperative approaches over non-cooperative alternatives has, however, been repeatedly and convincingly demonstrated through the development and comparison of multiple algorithms [8], [23]-[25], and no argument to the contrary is made here.

We do emphasise, however, that previous efforts to obtain CRLB (see for instance [1] and [5], as well as references thereby) for distributed cooperative network localization systems failed to account for the uncertainty of node locations in the estimation process, which explains the typically loose comparison between analytical and empirical results. To elaborate, as briefly mentioned earlier, the result of such omission is that the derived bounds actually represent the achievable errors of centralized approaches, in which the location of all nodes are computed simultaneously without requiring iterative distribution of previous location estimates amongst nodes themselves.

\section{REFERENCES}

[1] K. Langendoen and N. Reijers, "Distributed Localization in Wireless Sensor Networks: a Quantitative Comparison," Comput. Netw., vol. 43, no. 4, pp. 499-518, 2003.
[2] F. Gustafsson and F. Gunnarsson, "Mobile Positioning using Wireless Networks: Possibilities and Fundamental Limitations based on Available Wireless Network Measurements," Signal Processing Magazine, IEEE, vol. 22, no. 4, pp. 41-53, July 2005.

[3] U. Ferner, H. Wymeersch, and M. Win, "Cooperative Anchor-less Localization for Large Dynamic Networks," in Ultra-Wideband, 2008. ICUWB 2008. IEEE International Conference on, vol. 2, Sept. 2008, pp. $181-185$.

[4] S. Severi, G. T. F. de Abreu, and D. Dardari, "Distributed localization: Multihop versus message-passing," in Proc. IEEE International Workshop on Systems, Signals and Image Processing (IWSSIP 2012), 2012.

[5] N. Patwari, J. Ash, S. Kyperountas, I. Hero, A.O., R. Moses, and N. Correal, "Locating the Nodes: Cooperative Localization in Wireless Sensor Networks," Signal Processing Magazine, IEEE, vol. 22, no. 4, pp. $54-69$, July 2005.

[6] Y. Shen, H. Wymeersch, and M. Z. Win, "Fundamental limits of wideband localization - part ii: Cooperative networks," IEEE Trans. Inform. Theory, vol. 56, no. 10, pp. 4981 - 5000, Oct. 2010.

[7] Y. Shen and M. Z. Win, "Fundamental limits of wideband localizationpart i: a general framework," Information Theory, IEEE Transactions on, vol. 56, no. 10, pp. 4956-4980, 2010.

[8] M. M.-B. M. Mofarreh-Bonab, "The effect of pruning stage in secure localization in wireless sensor networks,", in Sixth international Symposium on Telecommunications, Nov. 2012, pp. 455-458.

[9] F. Ghassemi and V. Krishnamurthy, "Decentralized node selection for localization in wireless unattended ground sensor networks," in Proc. IEEE International Conference on Sensor Technologies and Applications, 2008

[10] L. Liu, X. Zhang, and H. Ma, "Optimal node selection for target localization in wireless camera sensor networks," IEEE Trans. Vehicular Technology, vol. 59, no. 7, pp. 3562 - 3576, 2010.

[11] Y. Ding, N. Krislock, J. Qian, and H. Wolkowicz, "Sensor network localization, euclidean distance matrix completions, and graph realization." in MELT, 2008.

[12] J. Bruck, J. Gao, and A. A. Jiang, "Localization and routing in sensor networks by local angle information," in Proceedings of the 6th ACM International Symposium on Mobile Ad Hoc Networking and Computing, 2005.

[13] A. Basu, J. Gao, J. S. B. Mitchell, and G. Sabhnani, "Distributed localization using noisy distance and angle information," in Proceedings of the 7th ACM International Symposium on Mobile Ad Hoc Networking and Computing, 2006.

[14] A. K.-S. W. Robin Wentao Ouyang and C.-T. Lea, "Received signal strength-based wireless localization via semidefinite programming: Noncooperative and cooperative schemes," in IEEE Trans. Vehicular Technology, 2010.

[15] X. Li, "Collaborative localization with received-signal strength in wireless sensor networks," IEEE Trans. Vehicular Technology, 2007.

[16] E. Xu, Z. Ding, and S. Dasgupta, "Wireless source localization based on time of arrival measurement," in ICASSP, 2010.

[17] B. Yang and J. Scheuing, "Cramer-Rao bound and optimum sensor array for source localization from time differences of arrival," in Acoustics, Speech, and Signal Processing, 2005. Proceedings.(ICASSP'05). IEEE International Conference on, vol. 4. IEEE, 2005, pp. iv-961.

[18] T. Jia and R. M. Buehrer, "A new Cramer-Rao lower bound for TOA-based localization," in Military Communications Conference, 2008. MILCOM 2008. IEEE, nov. 2008, pp. 1 - 5.

[19] S. M. Kay, Fundamentals of Statistical signal processing, Volume 2: Detection theory. Prentice Hall PTR, 1998.

[20] D. R. Cox and D. V. Hinkley, Theoretical statistics. Chapman \& Hall/CRC, 1979.

[21] M. K. Thomas Hillebrandt, Heiko Will, Progress in Location-Based Services. Springer Berlin Heidelberg, 2013, no. pp 173-194.

[22] P. Tichavsky, C. Muravchik, and A. Nehorai, "Posterior cramer-rao bounds for discrete-time nonlinear filtering," IEEE Trans. on Signal Processing, 2005

[23] N.-H. Z. Leung and K.-C. Toh, "An SDP-based divide-and-conquer Algorithm for large Scale noisy Anchor-free Graph realization," 2008.

[24] A. Kannan, B. Fidan, G. Mao, and B. Anderson, "Analysis of Flip Ambiguities in Distributed Network Localization," Feb. 2007, pp. 193198.

[25] K. Heurtefeux and F. Valois, "Topology control algorithms: a qualitative study during the sensor networks life." in MASS, 2007. 